\title{
APNEA DEL RECIÉN NACIDO PRETÉRMINO
}

\author{
Héctor Romero MD*, Sabrina López MD**
}

\section{Resumen}

La apnea del recién nacido pretérmino es una condición con alto riesgo de morbimortalidad es este grupo etario, es por ello que pediatras, neonatólogos, enfermeras, terapeutas y personal en general quienes identifican y de una u otra manera intervienen en la prevención y manejo de esta patología, se ven en la necesidad de permanecer en continua actualización. Se hace una revisión de la literatura acerca de la definición, diagnóstico y tratamiento actual que se puede y debe ofrecer al recién nacido pretérmino con apnea.

Palabras clave: apnea, pretérmino, bradicardia, desaturación, diagnóstico, tratamiento.

Abreviaturas: RN, recién nacido; APNP, apnea del neonato prematuro.

\section{APNEA OF PREMATURITY}

\section{Abstract}

\begin{abstract}
Apnea in premature infants is a problem associated with high risk of morbidity and mortality in this age group. For this reason, pediatricians, neonatologists, nurses, care givers and pediatric general staff who identify, and in a way or another, intervene in preventing and managing this condition, need to continuously update. A literature review concerning, definition, diagnosis and available current treatment that must be offered to the premature infants with apnea was conducted.
\end{abstract}

Key words: apnea, preterm, bradycardia, desaturation, diagnostic, treatment

\section{Introduc ción}

La apnea del prematuro es una patología que conlleva una evidente morbimortalidad, se reconoce un escenario complejo ya que hay variabilidad en el nivel de entrenamiento y de desempeño de diferentes profesionales y sistemas de salud en su capacidad para detectarla en forma oportuna y correcta. Esta variabi- lidad justifica la necesidad de generar guías basadas en evidencia que permitan la racionalización y optimización del recurso humano y técnico necesario para la detección y diagnóstico correcto y eficiente de la APNP.

La disponibilidad de una guía de práctica clínica para la detección y el seguimiento de esta afección implica

\footnotetext{
Fecha recibido: noviembre 27 de 2013 - Fecha aceptado: marzo 7 de 2014

* Pediatra. Instructor Asociado, Fundación Universitaria de Ciencias de la Salud. Bogotá DC. Colombia. 
generar mayor eficiencia, oportunidad y calidad en el sistema de salud colombiano, optimizar el recurso humano y técnico en el abordaje de esta patología e impactar a corto, mediano y largo plazo en los perfiles de morbimortalidad de la población infantil, apoyados en estrategias basadas en evidencia clínica.

Como es relevante conceptualizar este tema, se hace una revisión acerca de la definición y énfasis en manejo a través de una búsqueda basada en términos definidos por descriptores en ciencias de la salud (DECS) y Medical Subject Headings ( $M e S H)$, búsqueda realizada en bases de datos en inglés de PUBMED, HINARI, OVID y datos en español en LILACS y SCIELO. Se revisaron 50 artículos en texto completo, los cuales se incluyen todos por ser recientes y de relevancia clínica.

\section{Antecedentes}

Las principales causas de muerte neonatal en el mundo son los nacimientos pretérmino (28\%), infecciones severas (36\%, incluyendo sepsis/neumonía $26 \%$, tétano $7 \%$ y diarrea $3 \%$ ) y complicaciones de la asfixia (23\%), todas relacionadas con los trastornos respiratorios neonatales. Según datos de la OMS, en Colombia la mortalidad neonatal es de 14 por cada 1.000 nacidos vivos y la perinatal (mortinatos y mortalidad neonatal temprana, primeros siete días de vida) es 23 por cada 1.000 nacidos vivos. $^{1}$

La incidencia de la APNP se correlaciona de manera inversa con la edad gestacional y el peso al nacer. El $7 \%$ se presenta entre las 34 y 35 semanas de gestación, el $15 \%$ a las 32 a 33, el $54 \%$ a los 30 a 31 semanas $^{2}$ y casi todos los bebés nacidos con menos de 29 semanas de gestación o <1.000 gramos la desarrollaron. ${ }^{3}$ En general se divide en tres subtipos: obstructiva, central, o mixta. ${ }^{4}$ La central representa $10 \%$ a $25 \%$ de todos los casos de apnea, la obstructiva de $10 \%$ a $25 \%$ y la mixta de $50 \%$ a $75 \% .^{4}$

La incidencia de bradicardia es bastante similar en estos diferentes grupos, sin embargo parece ocurrir con más frecuencia y mayor duración que la apnea. La bradicardia se produce en el 10\% de los episodios de apnea con una duración de 10 a 14 segundos, el
$34 \%$ cuando es entre 15 y 20 segundos y el $75 \%$ si se prolonga por más de veinte segundos. ${ }^{5}$

\section{Definición}

La APNP es una manifestación de la inestabilidad del ritmo respiratorio, lo que refleja la inmadurez del sistema de control de las vías respiratorias. Desde el punto de vista anatómico la inmadurez se manifiesta como disminución de conexiones sinápticas, arborización dendrítica y pobre mielinización. La APNP se resuelve con la maduración, se debe considerar como un trastorno del desarrollo en lugar de un estado de enfermedad. ${ }^{6}$

La APNP corresponde a una pausa de la respiración por más de 15 a 20 segundos, acompañada de una desaturación de oxígeno $\left(\mathrm{SaO}_{2} \leq 80 \%\right.$ por más de 4 segundos) y bradicardia (frecuencia cardíaca $<2 / 3$ de línea de base durante $\geq 4$ segundos), en recién nacidos de menos de 37 semanas de gestación. ${ }^{2}$

Según la presencia o no de esfuerzos respiratorios se clasifican en centrales cuando hay cese de movimientos respiratorios y representan un 10-25\%, obstructivas si hay movimientos respiratorios pero no flujo aéreo (10-20\%) y mixtas ante obstrucción más pausas de movimientos respiratorios (50-75\%) que son las más comunes.

El sitio principal de la obstrucción de la vía aérea superior es la faringe, aunque también puede ocurrir en la laringe y es posible en ambos sitios. La apnea mixta por lo regular representa más del $50 \%$ de los episodios largos de apnea, seguida en orden decreciente de frecuencia por la central y la obstructiva. La obstructiva espontánea pura en ausencia de un problema posicional es un episodio raro. ${ }^{7}$

Mientras la APNA es un trastorno del desarrollo, las razones de la tendencia para que ocurra en recién nacidos inmaduros no son del todo claras. Aunque la patogenia es poco conocida, los reflejos inmaduros pulmonares y respiratorios y la respuesta a la hipoxia y la hipercapnia es posible que contribuyan a la aparición o a la gravedad de la APNP. ${ }^{8}$ También puede 
exacerbarse por una serie de factores coexistentes o estados de enfermedad. ${ }^{9}$ Los mecanismos reconocidos se analizan a continuación:

Transición fetal a neonatal: el feto se encuentra en un entorno pobre de oxígeno, con $\mathrm{PaO}_{2}$ de 23 a $27 \mathrm{~mm} \mathrm{Hg}$. Tras el nacimiento pasa a un ambiente rico en oxígeno con una $\mathrm{PaO}_{2}$ cuatro veces mayor. ${ }^{10}$ Ese aumento en la $\mathrm{PaO}_{2}$ inhibe los quimiorreceptores periféricos, dando lugar a la aparición tardía de la respiración espontánea, en especial cuando los RN están expuestos a oxígeno al $100 \%$ durante la reanimación postnatal. ${ }^{11}$ Por tal motivo los RN necesitan ajustar rápido su ventilación a la vida postnatal.

Respuesta ventilatoria a la hipoxia: después del nacimiento el prematuro presenta un aumento transitorio de la frecuencia respiratoria y del volumen corriente, seguido de una disminución sostenida de la respiración espontánea que puede durar varias semanas, a lo que se le denomina depresión respiratoria hipóxica que puede estar asociada con el ajuste respiratorio postnatal retardado. ${ }^{12}$ La estimulación de los quimioreceptores periféricos puede dar lugar a la hipocapnia secundaria vista después de la hiperventilación. ${ }^{13} \mathrm{El}$ nivel del $\mathrm{CO}_{2}$ puede disminuir cerca del umbral de apnea ( 1 a $1.3 \mathrm{~mm}$ $\mathrm{Hg}$ por debajo de la línea de base del nivel de $\mathrm{CO}_{2}$ ), que junto con la activación de los quimioreceptores en respuesta a la hiperventilación puede conducir a apnea. $^{14}$

Respuesta ventilatoria a la hipercapnia: en respuesta a la hipercapnia los prematuros aumentan la ventilación prolongando el periodo de cierre sin aumentar la frecuencia respiratoria o el volumen corriente, lo cual conduce a menor volumen minuto que lo observado en los $\mathrm{RN}$ a término. Los movimientos paradójicos de los músculos de las vías respiratorias en respuesta a la hipercapnia, también pueden jugar un papel importante en la apnea. ${ }^{15}$

La respuesta ventilatoria de la laringe al quimioreflejo: hace referencia a la activación que ocurre en la mucosa laríngea en prematuros conduciendo a apnea, bradicardia e hipotensión. ${ }^{16}$ Esta respuesta se considera un reflejo de protección, pero una exagerada puede producir apnea y se denomina quimiorreflejo laríngeo, mediado por las fibras aferentes del nervio laríngeo superior. ${ }^{17}$

Neurotransmisores y apnea: la sensibilidad aumentada a los neurotransmisores inhibitorios como GABA, serotonina, adenosina y prostaglandina es característica del sistema de control respiratorio del prematuro. ${ }^{18}$ Durante muchos años la investigación se ha centrado en la localización de sitios quimiosensoriales. Estos estudios demuestran que los elementos neurales sensibles a la quimioterapia de la superficie ventrolateral de la médula, juegan un papel fundamental en la regulación de la actividad respiratoria y la respuesta ventilatoria de $\mathrm{CO}_{2}$. Datos fisiológicos recientes revelan la presencia de sitios sensibles a la quimioterapia en las regiones fuera del área ventrolateral del bulbo raquídeo. ${ }^{19}$

Variabilidad genética y apnea: estudios recientes encontraron la relación de herencia con apnea en un $87 \%$ entre gemelos del mismo sexo, lo que plantea una base genética importante. ${ }^{20}$ La apnea se presenta con más frecuencia en el sueño REM que en el sueño más tranquilo. El despertar del REM parece ser un precursor de la apnea asociada con desaturación de oxígeno, ya que es típico que las actividades del centro después de la excitación se asocien con el cierre laríngeo. ${ }^{21}$ Además, neurotransmisores y neuromoduladores, incluyendo dopamina, adenosina, endorfinas, GABA y prostaglandinas, han sido implicados en la patogénesis de los trastornos de la respiración, sustancias que pueden alterarse en los primeros años de vida. ${ }^{22}$

Si bien el control respiratorio inmaduro es la principal causa de APNP, muchos factores coexistentes pueden potenciarla o empeorarla. Se presenta tanto en infección local como sistémica ${ }^{23}$ y puede activarse por enfermedades del sistema nervioso central incluyendo hemorragia intracraneal, encefalopatía hipóxico-isquémica y convulsiones. La termorregulación también puede desempeñar un importante papel en la apnea, porque la exposición a temperaturas bajas disminuye la duración y frecuencia de apnea, mientras que la temperatura elevada aumenta la incidencia, lo que sugiere relación con el estado metabólico y la tempe- 
ratura ambiente..$^{24}$ Otros factores que se han asociado incluyen alteración metabólica de la glucosa, desequilibrio electrolítico ${ }^{4}$ y la presencia de DAP con corto circuitos grandes. ${ }^{25} \mathrm{La}$ anemia también se asocia debido a la hipoxia que lleva a depresión respiratoria. El reflujo gastroesofágico no parece estar relacionado con la aparición de apnea, aunque algunas condiciones pueden exacerbarlo. Sin embargo, no hay evidencia que apoye el uso de medicamentos antirreflujo para el tratamiento de la APNP. ${ }^{26}$ Otro factor es la flexión del cuello, porque influye en la regulación neuromuscular de la permeabilidad de la faringe y puede producir obstrucción intermitente de la vía aérea. ${ }^{27}$ La obstrucción nasal por edema o presencia de sonda nasogástrica aumenta la resistencia de la vía aérea y el vaciamiento gástrico retardado puede aumentar los episodios de apnea porque la distensión abdominal reduce el volumen pulmonar y aumenta la retroalimentación aferente vagal. ${ }^{28}$

\section{Epidemiología}

La incidencia de la apnea aumenta con la disminución de la edad gestacional. En un estudio multicéntrico fue diagnosticada en $50 \%$ de los bebés nacidos entre las 33 y 34 semanas de gestación según la documentación de la historia clínica ${ }^{29}$ y ocurre en casi todos los nacidos cuando la gestación es menor de 28 semanas. ${ }^{30,31}$ En gestación de 24 a 28 semanas puede ocurrir apnea postconcepcional hasta la semana 38 , lo que prolonga la hospitalización. ${ }^{30}$ La resolución suele ser completa alrededor de las 43 a 44 semanas de edad gestacional corregida (PMA) ${ }^{32}$ La situación guarda relación inversamente proporcional con la edad gestacional, como se evidencia en la Tabla $\mathbf{1 . 3}^{33}$

\begin{tabular}{|c|c|}
\hline \multicolumn{2}{|c|}{ Tabla I. Edad gestacional y frecuencia } \\
\hline Edad gestacional en semanas & Frecuencia de apnea \\
\hline 34 a 35 & $7 \%$ \\
\hline 32 a 33 & $15 \%$ \\
\hline 30 a 31 & $54 \%$ \\
\hline Menos de 29 & Casi $100 \%$ \\
\hline
\end{tabular}

Fuente: Precop año 5 módulo I.Apnea de la prematurez.

Los estudios muestran que en la raza blanca y los niveles previos de bilirrubina por encima de $10 \mathrm{mg} / \mathrm{dL}$, se asocian desde el punto de vista estadístico con la persistencia de apnea. Además, la hiperbilirrubinemia puede producir efectos deletéreos persistentes en el cerebro medio de los $\mathrm{RN}$ de 36,6 semanas de edad postconcepcional y afectar en forma adversa el control de la respiración. ${ }^{33}$

\section{Diagnóstico}

Respiración: los monitores cardiorrespiratorios neonatales utilizan la tecnología de impedancia para medir la respiración, que sólo corresponde al movimiento de la pared torácica. Estos son útiles sólo para diagnosticar la apnea central y el uso en apnea mixta no es efectivo, pues suele asociarse con cierre de la vía aérea superior y la actividad muscular respiratoria continua. El monitor posee alarmas para identificar un cese de la respiración durante 15 a 20 segundos.

Frecuencia cardíaca: el monitoreo es importante en la detección de la APNP por la alta frecuencia de bradicardia. Se considera que es una respuesta refleja a la caída en la oxigenación. La razón más frecuente para las alarmas cardiorrespiratorias en prematuros es la ocurrencia de pausas breves $(<15$ segundos) asociadas con bradicardia. ${ }^{34}$

Oximetría de pulso: se utiliza como un complemento frecuente en los monitores cardiorrespiratorios en la unidad de cuidado intensivo neonatal. Al igual que con las alarmas de bradicardia, proporciona una medida de seguridad adicional que se ajusta a $<85$ o $<80$ saturación de oxígeno. La incidencia y la profundidad de los episodios de desaturación de oxígeno después de la apnea son dependientes de las tiendas de oxígeno pulmonar y el nivel de los niveles de saturación de oxígeno de referencia. ${ }^{35}$ Muchos RN prematuros tienen volúmenes pulmonares bajos asociados con la enfermedad pulmonar residual y una pared toráxica obediente, son vulnerables a caídas frecuentes y profundas en la saturación de oxígeno. El intervalo de tiempo para la determinación de la saturación media de oxígeno se puede ajustar y se suavizará la señal para disminuir el número de alarmas, aunque no detectará períodos cortos de desaturación de oxígeno. ${ }^{36}$ 


\section{Tratamiento}

Muchas veces estímulos externos resultan suficientes para que el niño reinicie los movimientos respiratorios. Si no es así, se asiste la ventilación con oxígeno mediante ventilación con bolsa-válvula-mascarilla (resucitador manual).$^{37}$ Calidad de evidencia: moderada.

A menudo al aplicar la asistencia ventilatoria con resucitador manual se distiende el estómago con el peligro posterior de vómito y broncoaspiración, por lo que conviene vaciarlo a través de una sonda orogástrica y dejar al niño en ayuno mientras se realizan estas maniobras. Deben vigilarse con frecuencia los signos vitales por medios electrónicos (pulsoximetría), con el estetoscopio o bien observando el color de la piel, tono y reflejos. ${ }^{38}$ Calidad de evidencia: alta.

Se promueven aquellas que la evitan a través de la estimulación cutánea y vestibular como hacerlo llorar con frecuencia, tocarlo, moverlo, pequeños golpecitos en el cuerpo; el uso de la estimulación a base de movimientos (colchón con agua en constante movimiento, etc.) no ha mostrado ser un tratamiento útil para prevenir la APNP. ${ }^{37}$ Calidad de evidencia: baja.

La posición más aconsejable hasta el momento y con menos riesgo para dormir es la supina. ${ }^{39-41}$ Calidad de la evidencia: baja.

Si no se descubre una causa específica, el diagnóstico es de APNP por inmadurez y el problema puede ceder con las medidas generales antes expuestas. Pero debido a que es muy frecuente que se vuelvan a presentar, es recomendable el uso de metilxantinas ${ }^{42}$ para su tratamiento (el citrato de cafeína). ${ }^{43-45}$ Calidad de la evidencia: alta Como primera elección con dosis de impregnación de $20 \mathrm{mg} / \mathrm{k}$ de peso $(10 \mathrm{mg} / \mathrm{k}$ de cafeína base) intravenosa seguida de $5 \mathrm{mg} / \mathrm{k} /$ día ( $2.5 \mathrm{mg} / \mathrm{k} /$ día de cafeína base) antes que la teofilina, ya que tiene menos efectos adversos, su vida media es más amplia y sus niveles plasmáticos son más estables. Ambas tienen el mismo efecto terapéuti$\mathrm{co}^{45}$, el inconveniente es que en nuestro país es difícil conseguirla, por lo que ante esta situación se debe escoger entonces como primera elección la teofilina antes de utilizar la presión positiva continua de la vías respiratorias (PPCVR).

Debe recordarse que las xantinas nunca se emplean para tratar la apnea asociada con una causa bien definida y sólo se reserva para neonatos en quienes se excluye una causa corregible de apnea. Los efectos secundarios con la teofilina son irritabilidad, insomnio, temblores, taquicardia y otras arritmias cardíacas, hipo o hipertensión, dolor abdominal, anorexia, náuseas, vómito, hematemesis, deshidratación albuminuria y convulsiones. ${ }^{46}$

La presión positiva intermitente de las vías respiratorias (PPIVR) mediante puntas nasales puede ser un método útil ya que parece reducir la frecuencia de apneas en forma más efectiva que la PPCVR, solo que se requieren más estudios para valorar su eficacia y seguridad, antes de recomendarla como terapéutica estándar. ${ }^{47}$ Calidad de la evidencia: alta

\section{Otras terapias}

Transfusión: La frecuencia y la gravedad de la apnea se incrementan en los prematuros que desarrollan anemia significativa en uno a dos meses de edad. Parece que la transfusión reduce la frecuencia de la apnea. ${ }^{48}$

Plan de salida: la monitorización cardiorrespiratoria en casa no es necesario para los niños que permanecen libres de cualquier episodio de apnea, bradicardia o desaturación de oxígeno durante cinco a siete días, ya que el riesgo de un posterior evento de apnea con significación clínica es muy baja. ${ }^{49}$ Sin embargo, si el prematuro está listo para la salida pero la apnea leve sigue siendo una preocupación, es incierto cuál es el enfoque óptimo. Muchos tienen bradicardia leve persistente y/o episodios de desaturación que son detectados por monitorización cardiorrespiratoria. Si la cafeína ha sido descontinuada hace poco, por lo general, se recomienda no dar de alta a menos que haya un período libre de eventos de siete (en lugar de cinco) días para permitir que la cafeína se elimine o llegue a niveles subterapéuticos bajos. ${ }^{50}$ 


\section{Referencias}

1. Zuñiga M. Informe del evento de mortalidad perinatal y neonatal tardia, hasta e periodo epídemiologico 12 del año 2012. Bogotá: Instituto Nacional de Salud; 2012

2. Zhao J, Gonzalez F, Mu D. Apnea of prematurity: from cause to treatment. Eur J Pediatr. 2011 Sep;170(9):1097-105.

3. Robertson CM, Watt MJ, Dinu IA. Outcomes for the extremely premature infant: what is new? And where are we going?. Pediatr Neurol. 2009;40(3):189-96.

4. Stokowski LA. A primer on Apnea of prematurity. Adv Neonatal Care. 2005;5(3):155-70

5. Poets CF. Apnea of prematurity: What can observational studies tell us about pathophysiology?. Sleep Med. 2010;11(7):701-7.

6. Mathew OP. Apnea of prematurity: pathogenesis and management strategies. J Perinatol. 2011;31(5):302-10.

7. Martin RJ, Abu-Shaweesh JM, Baird TM. Pathophysiologic mechanisms underlying apnea of prematurity. NeoReviews. 2002;3(4):e59-e65.

8. Darnall RA. The role of $\mathrm{CO}(2)$ and central chemoreception in the control of breathing in the fetus and the neonate. Respir Physiol Neurobiol. 2010;173(3):201-12.

9. Baird TM. Clinical correlates, natural history and outcome of neonatal apnoea Semin Neonatol. 2004;9(3):205-11.

10. Mercer JS, Erickson-Owens DA, Graves B, Haley MM. Evidence-based practices for the fetal to newborn transition. J Midwifery Womens Health. 2007;52(3):262-72.

11. Vento M, Asensi M, Sastre J, Lloret A, Garcia-Sala F, Vina J. Oxidative stress in asphyxiated term infants resuscitated with $100 \%$ oxygen. J Pediatr. 2003;142(3):240-6.

12. Gauda EB, McLemore GL, Tolosa J, Marston-Nelson J, Kwak D. Maturation of peripheral arterial chemoreceptors in relation to neonatal apnoea. Semin Neonatol. 2004;9(3):181-94.

13. Cardot V, Chardon K, Tourneux P, Micallef S, Stephan E, Leke A, et al. Ventilatory response to a hyperoxic test is related to the frequency of short apneic episodes in late preterm neonates. Pediatr Res. 2007;62(5):591-6.

14. Khan A, Qurashi M, Kwiatkowski K, Cates D, Rigatto H. Measurement of the $\mathrm{CO} 2$ apneic threshold in newborn infants: possible relevance for periodic breathing and apnea. J Appl Physiol. 2005; 98(4):1171-6.

15. Carlo WA, Martin RJ, Difiore JM. Differences in $\mathrm{CO} 2$ threshold of respiratory muscles in preterm infants. J Appl Physiol. 1988;65(6):2434-9.

16. Praud JP. Upper airway reflexes in response to gastric reflux. Paediatr Respir Rev. 2010;11(4):208-12.

17. Kelly BN, Huckabee ML, Jones RD, Frampton CM. Nutritive and non-nutritive swallowing apnea duration in term infants: implications for neural control mechanisms. Respir Physiol Neurobiol. 2006;154(3):372-8.

18. Martin RJ, Abu-Shaweesh JM, Baird TM. Apnoea of prematurity. Paediatr Respir Rev. 2004;5 Suppl A:S377-82.

19. Dreshaj IA, Haxhiu MA, Martin RJ. Role of the medullary raphe nuclei in the respiratory response to CO2. Respir Physiol. 1998;111(1):15-23.

20. Bloch-Salisbury E, Hall MH, Sharma P, Boyd T, Bednarek F, Paydarfar D. Heritability of apnea of prematurity: a retrospective twin study. Pediatrics. 2010;126(4):e779-87.

21. Lehtonen L, Martin RJ. Ontogeny of sleep and awake states in relation to breathing in preterm infants. Semin Neonatol. 2004;9(3):229-38.

22. Gerhardt T, Bancalari E. Apnea of prematurity: I. Lung function and regulation of breathing. Pediatrics. 1984;74(1):58-62.

23. Kamaluddeen M, Lodha A, Akierman A. Non-Rotavirus infection causing apnea in a neonate. Indian J Pediatr. 2009;76(10):1051-2.

24. Tourneux P, Cardot V, Museux N, Chardon K, Leke A, Telliez F, et al. Influence of thermal drive on central sleep apnea in the preterm neonate. Sleep. 2008;31(4):549-56

25. Naik-Mathuria B, Chang S, Fitch ME, Westhoff J, Brandt ML, Ayres NA, et al. Patent ductus arteriosus ligation in neonates: preoperative predictors of poor postoperative outcomes. J Pediatr Surg. 2008;43(6):1100-5.
26. Tipnis NA, Tipnis SM. Controversies in the treatment of gastroesophageal reflux disease in preterm infants. Clin Perinatol. 2009;36(1):153-64.

27. Vialet R, Nau A. Effect of head posture on pediatric oropharyngeal structures: implications for airway management in infants and children. Curr Opin Anaesthesiol. 2009;22(3):396-9.

28. Thompson AM, Bizzarro MJ. Necrotizing enterocolitis in newborns: pathogenesis, prevention and management. Drugs. 2008;68(9):1227-38.

29. Eichenwald EC, Zupancic JA, Mao WY, Richardson DK, McCormick MC, Escobar GJ. Variation in diagnosis of apnea in moderately preterm infants predicts length of stay. Pediatrics. 2011;127(1):e53-8.

30. Eichenwald EC, Aina A, Stark AR. Apnea frequently persists beyond term gestation in infants delivered at 24 to 28 weeks. Pediatrics. 1997;100(3 Pt 1):354-9.

31. Hofstetter AO, Legnevall L, Herlenius E, Katz-Salamon M. Cardiorespiratory development in extremely preterm infants: vulnerability to infection and persistence of events beyond term-equivalent age. Acta Paediatr. 2008;97(3):285-92.

32. Ramanathan R, Corwin MJ, Hunt CE, Lister G, Tinsley LR, Baird T, et al. Cardiorespiratory events recorded on home monitors: Comparison of healthy infants with those at increased risk for SIDS. JAMA. 2001;285(17):2199-207.

33. Baquero Latorre H. Apnea de la prematurez. PRECOP. 2006;5(1):27-37.

34. Di Fiore JM, Arko MK, Miller MJ, Krauss A, Betkerur A, Zadell A, et al. Cardiorespiratory events in preterm infants referred for apnea monitoring studies. Pediatrics. 2001;108(6):1304-8.

35. Di Fiore JM, Walsh M, Wrage L, Rich W, Finer N, Carlo WA, et al. Low oxygen saturation target range is associated with increased incidence of intermittent hypoxemia. J Pediatr. 2012;161(6):1047-52.

36. Di Fiore JM, Bloom JN, Orge F, Schutt A, Schluchter M, Cheruvu VK, et al. A higher incidence of intermittent hypoxemic episodes is associated with severe retinopathy of prematurity. J Pediatr. 2010;157(1):69-73.

37. Osborn DA, Henderson-Smart DJ. Kinesthetic stimulation for treating apnea in preterm infants. Cochrane Database Syst Rev. 2000(2):CD000499.

38. Henderson-Smart DJ, Osborn DA. Kinesthetic stimulation for preventing apnea in preterm infants. Cochrane Database Syst Rev. 2000(2):CD000373.

39. Changing concepts of sudden infant death syndrome: implications for infant sleeping environment and sleep position. American Academy of Pediatrics. Task Force on Infant Sleep Position and Sudden Infant Death Syndrome. Pediatrics. 2000;105(3 Pt 1):650-6.

40. Jeffery HE, Megevand A, Page H. Why the prone position is a risk factor for sudden infant death syndrome. Pediatrics. 1999;104(2 Pt 1):263-9.

41. Keene DJ, Wimmer JE, Jr., Mathew OP. Does supine positioning increase apnea, bradycardia, and desaturation in preterm infants?. J Perinatol. 2000;20(1):17-20.

42. Henderson-Smart DJ, Steer P. Methylxanthine treatment for apnea in preterm infants. Cochrane Database Syst Rev. 2000(2):CD000140.

43. Comer AM, Perry CM, Figgitt DP. Caffeine citrate: a review of its use in apnoea of prematurity. Paediatr drugs. 2001;3(1):61-79.

44. Erenberg A, Leff RD, Haack DG, Mosdell KW, Hicks GM, Wynne BA. Caffeine citrate for the treatment of apnea of prematurity: a double-blind, placebo-controlled study. Pharmacotherapy. 2000;20(6):644-52.

45. Steer PA, Henderson-Smart DJ. Caffeine versus theophylline for apnea in preterm infants. Cochrane Database Syst Rev. 2000(2):CD000273.

46. American Academy of Pediatrics Committee on Drugs: Precautions concerning the use of theophylline. Pediatrics. 1992;89(4 Pt 2):781-3.

47. Hospital Británico. Departamento de Pediatría. Unidad Neonatal. Apnea del prematuro. Archiv Pediatr Urug. 2007;78:54-7.

48. Zagol K, Lake DE, Vergales B, Moorman ME, Paget-Brown A, Lee H, et al. Anemia, apnea of prematurity, and blood transfusions. J Pediatr. 2012;161(3):417-21 e1.

49. Lorch SA, Srinivasan L, Escobar GJ. Epidemiology of apnea and bradycardia resolution in premature infants. Pediatrics. 2011;128(2):e366-73.

50. American Academy of Pediatrics. Committee on Fetus and Newborn. Apnea, sudden infant death syndrome, and home monitoring. Pediatrics. 2003;111(4 Pt 1):914-7. 